\title{
EVALUACIÓN DE LA PERCEPCIÓN DE CONGESTIÓN DEL TURISTA EN EL CENTRO HISTÓRICO DE QUITO
}

\author{
Pablo Torres Matovelle* \\ Universidad de la Habana \\ Jesús Tapia López** \\ Universidad Central del Ecuador \\ Karla Suquilanda Agila***
}

\section{RESUMEN}

La congestión es un fenómeno que preocupa desde hace décadas a los gestores de destinos turísticos. Pocos estudios se han desarrollado para evaluarla en ciudades patrimoniales sobre todo a partir de la percepción del turista, notando un escaso tratamiento del tema en Latinoamérica. Esta investigación evalúa la percepción de congestión del turista en el Centro Histórico de Quito, mediante aportes metodológicos que vinculan variables de tipo motivacional, emocional y conductual. Los resultados indican que la percepción de congestión es percibida mayoritariamente entre leve y moderada.

Palabras clave: hacinamiento percibido, ciudad histórica, patrimonio de la humanidad, turismo, motivación.

\section{Tourist's crowding perception assessment in Quito's Historical Center}

\section{ABSTRACT}

Crowding is a phenomenon that has worried managers of tourist destinations for decades. Few studies have been developed to evaluate it in heritage cities from the perception of the visitor, noticing a poor treatment of the subject in Latin America. This research evaluates

Fecha de recepción: 16 de enero de 2018

Fecha de aceptación: 17 de septiembre de 2018

* Doctorante. Facultad de Turismo. Universidad de la Habana 506 G St. Vedado, CIUDAD DE LA HABANA (Cuba).E-mail: pablotorresmatovelle@gmail.com

**Facultad de Ciencias Administrativas. Universidad Central del Ecuador. Av. América y Pérez Guerrero. QUITO (Ecuador).E-mail: jtapial81@ hotmail.com

***Investigadora independiente. Mariscal Sucre OE6D QUITO (Ecuador).E-mail:Ksuquilanda@outlook.com 
the tourist crowding perception in the Historic Center of Quito, through methodological contributions that link motivational, emotional and behavioral variables. The results indicate that crowding is perceived mostly between mild and moderate.

Keywords: perceived crowding, historic city, world heritage, tourism, motivation.

\section{INTRODUCCIÓN}

Como lo refieren Torres y Villacís (2016), las ciudades del patrimonio mundial representan destinos altamente posicionados en rankings de comunidades virtuales especializadas en viajes, revistas electrónicas, redes sociales y concentradoras de imágenes de libre acceso de la WEB 3.0. Esto reafirma la situación de estas ciudades como casos atípicos en el ciclo del producto turístico, pues resisten entrar en la curva de declive (Russo, 2002), gracias al valor excepcional universal que las caracteriza y el poder atractor que este genera en la demanda.

La atracción de los destinos turísticos que gozan de la insignia de la UNESCO se da también en el campo académico. Así lo refieren Moreno, Pérez, Oyarce y López (2016) al señalar que la inscripción de un sitio como patrimonio de la humanidad y su relación con los flujos turísticos ha llegado a ser uno de los aspectos más analizados recientemente por los investigadores del turismo.

Para autores como Shia, Zhaob y Ju Chenc (2017), el auge del turismo urbano está coadyuvando al reconocimiento de la "congestión" como una preocupación social en muchas ciudades. Acotan no obstante, que la forma en que el turista experimenta la congestión sigue siendo todavía un tópico relegado en la investigación turística. De hecho, es notoria la escasez de aportes al tema en ciudades del patrimonio de la humanidad, especialmente en América. Por esta razón, este estudio tiene por objetivo evaluar la percepción de congestión de los turistas del Centro Histórico de la ciudad de Quito.

La identificación de variables influyentes en la percepción de congestión resulta un aspecto clave para orientar la gestión de flujos turísticos en destinos patrimoniales. Esto es especialmente importante en aquellos que muestran graves problemas de saturación y congestión como las Ciudades Patrimonio de la Humanidad (González, 2013).

El estudio ha seleccionado un método de corte cuantitativo, que parte de una encuesta aplicada a 800 turistas nacionales y extranjeros en dos de los sitios de mayor afluencia turística del Centro Histórico de Quito. El procesamiento de la información resultante se realiza mediante un modelo de análisis multivariado que incluyó análisis de componentes principales y regresión logística binaria.

El trabajo expone una descripción sintética del área de estudio y la revisión teórica en torno a las ciudades patrimoniales como destinos turísticos. Posteriormente refiere la percepción de congestión del turista como objeto de investigación. Acto seguido expone en detalle el proceso metodológico utilizado para la aplicación, procesamiento de la encuesta e interpretación de resultados. Finalmente presenta los resultados obtenidos con la discusión del alcance y connotación del estudio, así como las conclusiones. 


\subsection{Marco teórico}

\subsubsection{Descripción del área de estudio: El Centro Histórico de Quito (CHQ)}

La ciudad de San Francisco de Quito, fue fundada por españoles en 1534, constituyéndose en la capital más antigua de la América Meridional (López, 2005). La ciudad en su proceso histórico múltiple y diverso, tiene un antecedente pre-incaico como centro de articulación de pequeños poblados denominados "señoríos". Con la presencia Inca, Quito pasa a convertirse en una nueva centralidad política, administrativa y ritual de los Incas, similar a Cusco; y, con la conquista española, adquiere un rol de centralidad cristiana (Municipio DMQ, 2003). A finales del siglo XX, el desarrollo de la ciudad determina un salto a su condición actual de área metropolitana con múltiples centralidades.

El Distrito Metropolitano de Quito (DMQ) ocupa una superficie de 4.235,2 $\mathrm{km}^{2}$ y alberga al 15,8\% de la población del Ecuador. Representa la capital política-administrativa del país y mantiene un territorio organizado en ocho administraciones zonales que contienen a sesenta y cinco parroquias, de las cuales treinta y dos son urbanas y treinta y tres rurales (Municipio DMQ, 2011).

El DMQ contiene un Centro Histórico $(\mathrm{CH})$ cargado de valor político, simbólico y económico, que fue declarado Patrimonio Cultural de la Humanidad en 1978, siendo la primera ciudad en recibir tal distinción de la UNESCO.

El Centro Histórico cubre una superficie de 230 hectáreas de protección natural y 376 hectáreas de protección edificada. Esta última comprende 4 zonas: núcleo central, envolvente oriental, envolvente occidental y eje 24 de Mayo. El núcleo central, comprendido entre las calles Imbabura, Montúfar, Manabí y Av. 24 de Mayo, concentra, alrededor de la Plaza de la Independencia y otras áreas próximas, el principal espacio de uso turístico del Centro Histórico.

\section{Figura 1 \\ ZONAS DEL CENTRO HISTÓRICO DE QUITO Y SITIOS DE VISITA ICÓNICOS}

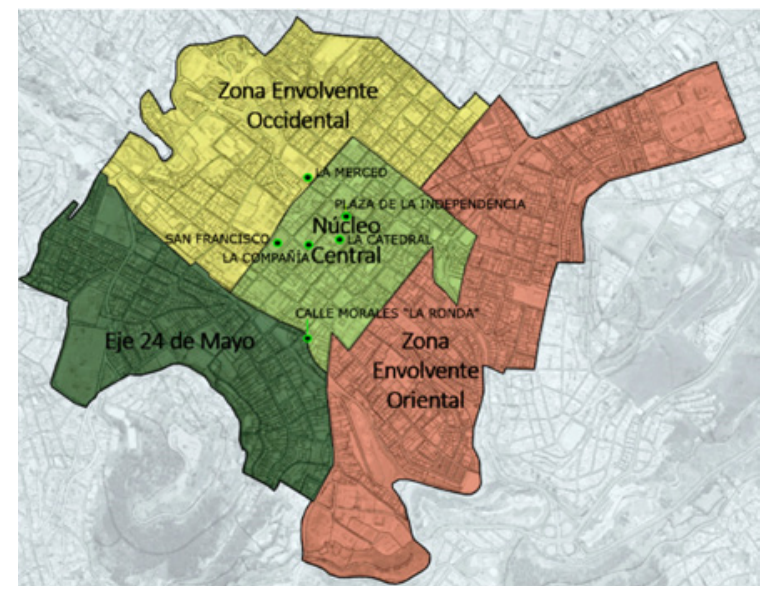

Fuente: Adaptado del mapa de zonas del Centro Histórico de Quito. Instituto de la Ciudad, 2018 
El CH constituye el principal atractivo turístico de la ciudad, así lo refieren el 67,2\% de los turistas extranjeros que lo visitan (EPMGDT, 2015). Cuentan entre sus sitios de visita icónicos: la Plaza de la Independencia, la calle La Ronda y las iglesias: San Francisco, La Compañía de Jesús, La Catedral y La Merced. La figura 1 muestra las zonas del Centro Histórico de Quito y la ubicación de los sitios de visita icónicos.

\subsubsection{La congestión en las ciudades históricas}

Como lo expone Brito (2008) toda ciudad es histórica por su propia existencia y trayectoria, no obstante, se reconoce como tal a aquella que posee un contenido histórico evidente, que contribuye al conocimiento y entendimiento del proceso civilizador de un pueblo y que además posee un carácter oficial fundamentado en el valor dado por la sociedad y el estado. De la Calle y García (2016), la define como una ciudad media de larga trayectoria, donde el patrimonio cultural constituye el núcleo de su identidad urbana, que en algunos casos por su valor universal excepcional ha logrado obtener la declaración de la UNESCO como Sitio Patrimonio de la Humanidad (Ruiz, 2012).

La incorporación del turismo en las ciudades antiguas provoca nuevos problemas relacionados con el equilibrio funcional, la gestión de flujos turísticos, la planificación urbanística, la protección del patrimonio y la accesibilidad, entre otros. Esto deja entrever que sin la aplicación de las medidas de ordenación oportunas, pueden producirse riesgos de importantes consecuencias (González, 2013). De la Calle y García (1998) citados por Cavaillès, Laurent, Maurin y Sánchez (2016), destacan dos problemas principales vinculantes: la excesiva concentración de visitantes en un número muy limitado de recursos y espacios, y la consecuente sub-utilización de un amplio conjunto de lugares alternativos.

Esto ha llevado a afirmar que las ciudades históricas pueden atraer con gran fuerza al turista, promoviendo la masificación con las consecuentes alteraciones al normal funcionamiento del espacio y afectando la calidad de la experiencia.

Desde el lado de la comunidad anfitriona también se reconocen efectos adversos. Doxey (1975) a través del modelo Irridex, que analiza las actitudes de los residentes, explica en cuatro etapas las reacciones de una comunidad residente frente al desarrollo turístico y el incremento de turistas: euforia - beneplácito, apatía, molestia y antagonismo (Brida, Riaño y Zapata, 2012).

Cabe también apuntar que la afluencia de turistas a las ciudades ha sido asociada al proceso de gentrificación turística, una de las manifestaciones del turismo más evidentes en destinos urbanos (García Herrera et al., 2007 citado por Benach y Albet, 2018). Delgadillo (2015) haciendo referencia al relevamiento de la vocación turística de los centros históricos europeos y latinoamericanos en las últimas décadas, destaca la proliferación de procesos de gentrificación propiciados por el protagonismo del turismo como estrategia bandera en procesos de regeneración urbana y recuperación de patrimonio histórico.

La gentrificación turística es considerada un agente inductor del cambio en las actividades propias de un lugar e incluso en el tipo de habitante. Además, crea un conjunto de interacciones permanentes a nivel material y simbólico entre turistas y residentes. En ese sentido, las prácticas socio espaciales de los turistas, como lo refieren Hiernaux y 
González (2015: 63), son "lo suficientemente fuertes sino agresivas como para generar una confrontación cotidiana entre ellos y los demás grupos de 'habitantes' de un centro histórico".

Coulomb (2008) refiere estos conflictos como impactos del turismo y destaca entre algunos de ellos: las tensiones entre pobladores y turistas por el uso y abuso del espacio público, la folklorización de expresiones culturales genuinas y, la desigualdad entre pobladores vinculados y marginados de la industria turística. Otros autores han referido impactos adicionales como el incremento de desechos (Cravidão et al., 2018), la especulación de inmuebles (Troitiño, 1998; Salmerón, 2009) y la disponibilidad de vivienda (Cortes, 2002).

\subsubsection{La percepción de congestión del turista como objeto de investigación}

El turismo en las ciudades es uno de los fenómenos estudiados con gran interés en el mundo académico. De hecho, como lo refiere Ashworth (2012), las ciudades proveen el escenario más importante de la actividad turística. Además, la necesidad de entender y estudiar de cerca la ciudad como elemento orgánico en las vidas de las personas se ha vuelto trascendental en nuestros días (World Tourism Organization, 2012).

Un aspecto crítico que llama la atención respecto al turismo en ciudades es la necesidad de manejar adecuadamente los flujos de visitantes. Esto con el fin de evitar conflictos de congestión y potenciar ventajas competitivas en el destino. De ahí que el manejo de visitantes sea contemplado en varios modelos de competitividad (Ritchie y Crouch, 2003; Jiménez y Aquino, 2012). De hecho, los estudios acerca de la congestión proveen claridad sobre como monitorear y manejar los flujos en sitios de significancia natural, cultural o histórica (Moyle y Croy, 2007, citados por Moyle et al., 2017) y de esta manera favorecen una experiencia satisfactoria.

La percepción de congestión como un factor clave que influye en la experiencia turística es reconocida por varios autores (Galí Espelt, 2008; Pedersen, 2002 citado por García y De la Calle, 2012; Barrer, Hernández y Balbuena, 2017; East, Osborne, Kemp y Woodfine, 2017). Adicionalmente, la percepción de congestión ha sido considerada un indicador para medir la capacidad de carga social en el contexto del turismo urbano (Almeida, 2006), sin embargo, como lo han referido García, De la Calle y Mínguez (2011: 222), "son pocos o casi inexistentes las publicaciones y trabajos que se pueden rastrear en relación a la aplicación del concepto a espacios turísticos de dominante históricopatrimonial".

La investigación sobre congestión, percepción de congestión y mecanismos para su manejo, comenzaron a principios de la década de 1970 (Nickerson, 2016). La congestión o hacinamiento percibido es un término que procede de la evaluación subjetiva de una persona respecto a la densidad en un entorno específico (Zhang \& Chung, 2015). Su carácter subjetivo da cuenta de la concurrencia de diversos factores psico-sociales, condicionados por la influencia de aspectos intrínsecos al individuo como la cultura, y extrínsecos vinculados al ambiente (Fleishman, Feitelson, y Salomon, 2004), como el tipo de turismo desarrollado y la temporalidad (Santana-Jiménez y Hernández, 2011)

Algunas investigaciones sobre la relación entre cultura/nacionalidad y percepción de hacinamiento revelan que los individuos con antecedentes culturales o étnicos similares, 
tienden a percibir el ambiente en patrones semejantes. Por tanto, la nacionalidad y la cultura asociada a esta, es un factor influyente tanto para la motivación como para la percepción de hacinamiento (Jin, Hui y Philip, 2016). Un ejemplo de ello puede notarse bajo la dimensión individualismo/colectivismo, que evalúa al individuo o al grupo como la unidad más importante (Neuts, Nijkamp y Van Leeuwen, 2012), y a partir de la cual se entiende como algunas culturas se muestran menos tolerantes y más sensibles a la congestión que otras.

De acuerdo con autores como Luque, Gómez y Peláez (2018), las motivaciones son factores altamente influyentes en la percepción de congestión. Por otra parte, Naoi, Airey, Iijima y Niininen (2005) señalan la divergencia existente en la connotación positiva o negativa que puede tener la percepción de congestión del turista. Pedersen (2002) señala que la congestión llega a ser un impacto negativo cuando interfiere con las expectativas de los turistas. Agrega que los efectos negativos varían con las características de los visitantes y su comportamiento, sus experiencias con el área y las peculiaridades del lugar.

Varios autores han investigado la relación de factores influyentes en la percepción de congestión. Entre ellos destacan: nivel de uso (Vaske y Donnelly, 2002); experiencias con el área y peculiaridades del lugar (Pedersen, 2002); normas individuales y sociales, tamaño de grupo y comportamiento de otros visitantes (Donnelly, Vaske, Whittaker y Shelby, 2000); duración de la estancia (Russo, 2002); motivaciones (Lee y Graefe, 2003; Jin et al., 2016); modo de viaje independiente u organizado (Kalisch y Klaphake, 2007); edad y nivel educativo (Rasoolimanesh et al., 2017); proximidad de los individuos (Szuster et al., 2011), disponibilidad general de recursos (Shelby, Vaske, y Heberlein, 1989); expectativas (Hui y Bateson, 1991); semejanza percibida con otros grupos y nacionalidad (Yagi y Pearce, 2007 citados por Neuts y Nijkamp, 2012).

Una variable que ha sido estudiada con menos profundidad en asociación al hacinamiento percibido por el turista es la emoción, la cual se considera como un indicador de la experiencia dentro del ítem multisensorialidad (Pulido y Navarro, 2014). Las emociones son representaciones experienciales de lo bueno o malo de algo referente (Martin y Clore, 2001 citados por Huang, Scott, Ding, y Cheng, 2012). Numerosos estudios se han conducido para explicar el rol de las emociones en diferentes aspectos del turismo y la hospitalidad pero escasamente para estudiar las dimensiones de la respuesta emocional del turista hacia el destino (Hosany y Gilbert, 2010). Varias propuestas se han llevado adelante para medir las emociones del turista en el contexto del consumo tales como el modelo placer-alerta-dominancia de Mehrabian y Russell, la escala de emoción diferencial de Izard, la teoría psico-evolucionaria de emociones de Plutchik, las escalas de afecto positivas y negativas de Watson, Clark y Tellegen y la escala de emoción de consumo de Richins (Hosany y Gilbert, 2010).

\section{METODOLOGÍA}

El estudio se llevó adelante en los dos sitios de espacio abierto más concurridos del Centro Histórico de Quito: la Plaza de la Independencia y la Calle La Ronda (Sistema de Indicadores Turísticos de Quito, 2016), como se aprecia en el cuadro 1. 


\section{Cuadro 1 \\ SITIOS MÁS VISITADOS DEL CHQ}

\begin{tabular}{|c|c|c|c|}
\hline Lugar & Turistas & $\%$ & Condición \\
\hline La Ronda & 21.635 & 51,19 & Espacio público abierto \\
Plaza de la Independencia & 1.131 & 2,68 & Espacio público abierto \\
Compañía de Jesús & 5.166 & 12,22 & Espacio privado confinado \\
La Catedral & 4.635 & 10,97 & Espacio privado confinado \\
San Francisco & 4.409 & 10,43 & Espacio privado confinado \\
La Basílica & 3.001 & 7.10 & Espacio privado confinado \\
Museo de la Ciudad & 2.289 & 5,42 & Espacio privado confinado \\
$\Sigma$ & 42.266 & 100 & \\
\hline
\end{tabular}

Fuente: Sistema de Indicadores Turísticos de Quito. Quito Turismo, 2016.

El cuestionario se estructuró en tres bloques tanto en idioma español como inglés. El primer bloque estuvo enfocado en el perfil socio-demográfico del turista e integró las variables: nacionalidad, género, edad, estado civil, formación académica, ocupación, acompañantes y duración de la estancia en Quito. Se plantearon 7 preguntas de opción múltiple cerradas y una pregunta abierta.

El segundo bloque estuvo orientado al perfil motivacional del turista e incorporó 26 motivaciones recuperadas e integradas de dos trabajos similares realizados en Brujas y Xian, ambas ciudades patrimonio de la humanidad (Jin et al., 2016; Neuts y Nijcamp, 2012). Para su valoración se aplicó una escala Likert de importancia de cinco puntos (desde nada importante hasta muy importante).

El tercer bloque dirigido a valorar la percepción de congestión, utilizó 4 preguntas: la primera para establecer el grado de congestión mediante una escala de 9 puntos ampliamente probada en estudios de este tipo (Shelby et al., 1989), donde el rango 1-2 refiere una percepción "no tan congestionado"; 3-5 "un poco congestionado"; 6-7 "moderadamente congestionado" y 8-9 "congestionado". La segunda pregunta se planteó para determinar la percepción positiva o negativa de congestión mediante una escala Likert de 5 puntos (desde muy negativa hasta muy positiva). La tercera pregunta se enfocó en evaluar la emoción respecto al grado de congestión percibida, utilizando igualmente una escala Likert de cinco puntos (desde totalmente en desacuerdo hasta totalmente de acuerdo). Se usó la batería de emociones propuesta por Plutchik (1980) que integra: alegría, sorpresa, admiración, coraje, tristeza, miedo y ansiedad. La batería ha sido aplicada en recientes estudios experienciales de turismo (Hernández, Vargasy Aguilar, 2015). La cuarta pregunta se formuló para determinar el grado de perturbación ocasionada por el comportamiento de los turistas en el sitio, específicamente acciones como: fumar en el espacio público, hacer ruido, pararse para tomar fotografías, chocar con otras personas, arrojar basura en la calle y obstaculizar el tráfico.

El cuestionario fue probado en un piloto con 16 encuestas que se aplicaron en cada uno de los dos lugares seleccionados. Se encontró el instrumento fiable de acuerdo con 
los valores calculados del Alpha de Cronbach: 0.84 en La Ronda y 0,82 en la Plaza de la Independencia. La aplicación se realizó entre el primero de junio y el primero de julio de 2017, obteniendo un total de 800 cuestionarios válidos, 400 en cada sitio de estudio. Los turistas fueron abordados en diferentes horarios, procurando lograr la representatividad de los periodos de mayor afluencia de personas. En la Plaza de la Independencia el horario de aplicación fue de 11:00 a 13:00 y de 15:00 a 15:30 todos los días. En La Ronda los turistas fueron encuestados los días viernes y sábados entre 19:00 y 20:30. Durante la aplicación del cuestionario se realizó paralelamente el conteo de personas presentes en los sitios de estudio. Para el caso de la Plaza de la Independencia, el conteo se realizó en el área de mayor congestión, es decir aquella contigua al monumento central de la estatua de La Libertad. Se delimitó un transecto semicircular alrededor del monumento, considerando exclusivamente el área de caminería disponible equivalente a 105,68 $\mathrm{m}^{2}$. Para el caso de la calle La Ronda, se delimitó un transecto de $100 \mathrm{~m}^{2}$ en la sección media de la calle Morales (La Ronda) entre las calles Guayaquil y Maldonado. Las figuras 2 y 3 muestran las áreas donde se realizó el conteo.

\section{Figura 2}

\section{TRANSECTO APLICADO EN LA PLAZA DE LA INDEPENDENCIA}

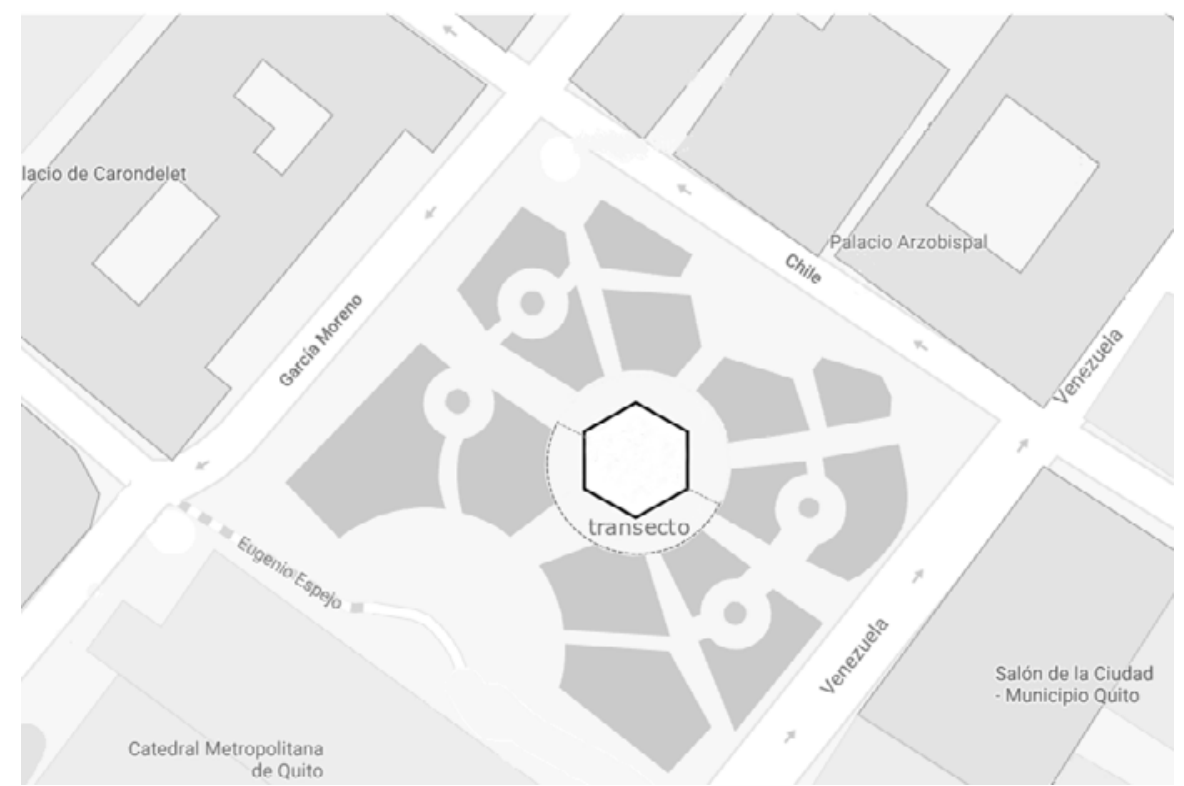

Fuente: adaptado de Google. (s.f.). [Mapa de Quito, Ecuador en Google maps]. Recuperado el 12 de Julio, 2018, de: https:/www.google.com.ec/maps/place/Plaza+Grande/@-0.2200615,-78.514263,17z/ data $=! 4 \mathrm{~m} 5 ! 3 \mathrm{~m} 4 ! 1 \mathrm{~s} 0 \times 91 \mathrm{~d} 5998$ 7820f190f:0x294cb69e1f4ca35e!8m2!3d-0.2200615!4d-78.5120744 


\section{Figura 3}

\section{TRANSECTO APLICADO EN LA CALLE LA RONDA}

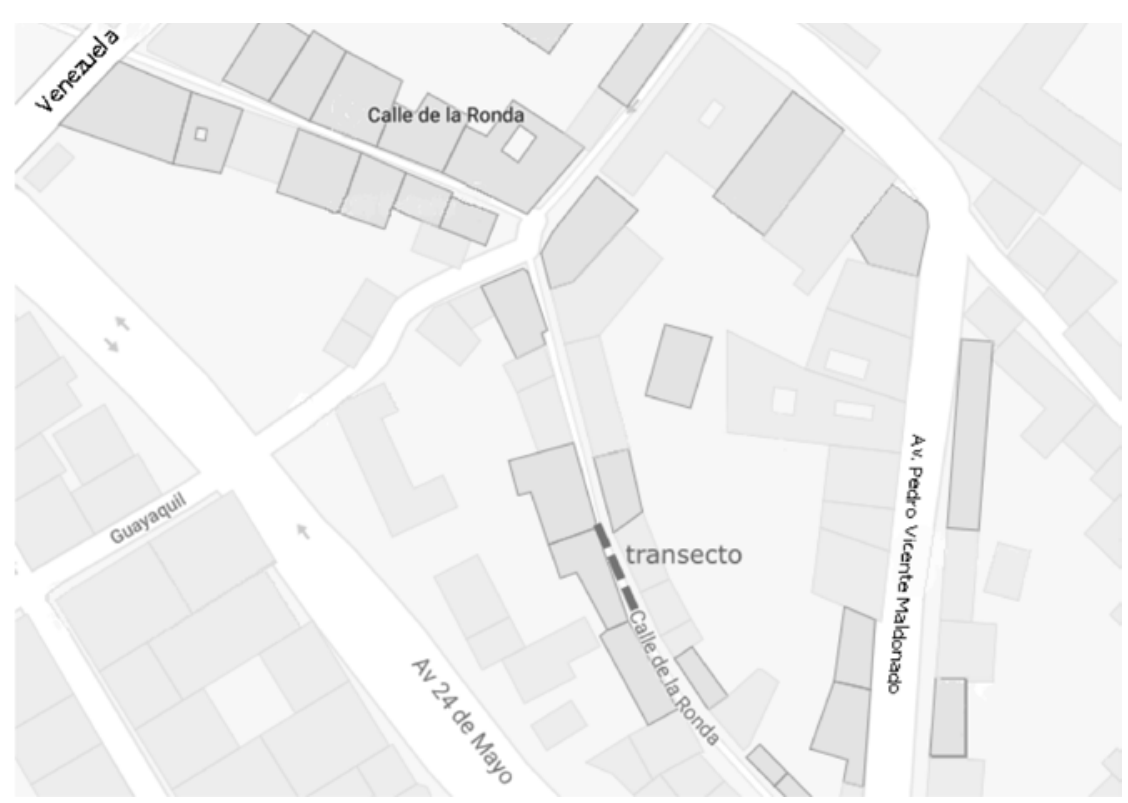

Fuente: adaptado de Google. (s.f.). [Mapa de Quito, Ecuador en Google maps]. Recuperado el 12 de Julio, 2018, de: https://www.google.com.ec/maps/search/Quito+la+ronda+calle+morales/@-0.2245258,-78.5161545,17z/ data $=! 3 \mathrm{~m} 1 ! 4 \mathrm{~b} 1$

El análisis estadístico aplicado fue descriptivo y multivariado. La revisión exploratoria permitió identificar posibles patrones de relacionamiento entre los factores que de acuerdo a la revisión bibliográfica influyen en la percepción de congestión. El análisis de componentes principales, sintetizó un número de seis factores, identificando asociaciones que en principio no se evidenciaron. Se realizó la prueba de Mann-Whitney para conocer la existencia o no de diferencias significativas en la percepción y valoración de la congestión entre los grupos encuestados en ambos sitios de estudio. Finalmente se utilizó un modelo de regresión logística binaria multivariante para identificar las variables que pueden explicar la percepción de congestión.

\section{RESULTADOS}

El perfil del encuestado describe un turista mayoritariamente extranjero del sexo femenino o masculino con menos de 45 años de edad. Se trata en la mayoría de los casos de un profesional preferentemente soltero o casado con estudios de nivel secundario y superior. Generalmente viaja en grupo y en menor medida en pareja, y permanece en Quito hasta 7 días, siendo lo más recurrente entre 1 y 3 días, como se puede ver en el cuadro 2. 
Cuadro 2

PERFIL DEL ENCUESTADO

\begin{tabular}{|c|c|c|c|c|c|c|c|}
\hline Variable & Categoría & Frecuencia & $\%$ & Variable & Categoría & Frecuencia & $\%$ \\
\hline \multirow[t]{3}{*}{ Sexo } & Masculino & 389 & 48,6 & \multirow[t]{3}{*}{ Estadía } & 1-3 días & 491 & 61,4 \\
\hline & Femenino & 411 & 51,4 & & 4-7 días & 229 & 28,6 \\
\hline & & & & & 8-11 días & 80 & 10,0 \\
\hline \multirow[t]{4}{*}{ Compañía } & Grupo & 482 & 60,3 & \multirow[t]{4}{*}{ Estudios } & Primaria & 7 & ,9 \\
\hline & Pareja o Compañero & 235 & 29,4 & & Secundaria & 300 & 37,5 \\
\hline & Solo & 83 & 10,4 & & Superior & 326 & 40,8 \\
\hline & & & & & Posgrado & 167 & 20,9 \\
\hline \multirow[t]{5}{*}{ Edad } & Menor de 25 años & 211 & 26,4 & \multirow[t]{5}{*}{ Estado civil } & Soltero & 312 & 39,0 \\
\hline & 26-35 años & 204 & 25,5 & & Casado & 256 & 32,0 \\
\hline & 36-45 años & 245 & 30,6 & & Divorciado & 71 & 8,9 \\
\hline & 46-65 años & 64 & 8,0 & & Unión Libre & 93 & 11,6 \\
\hline & 66 años en adelante & 76 & 9,5 & & Viudo & 68 & 8,5 \\
\hline \multirow[t]{6}{*}{ Nacionalidad } & Ecuador & 44 & 5,5 & \multirow[t]{6}{*}{ Ocupación } & Estudiante & 147 & 18,4 \\
\hline & $\begin{array}{l}\text { Otro país } \\
\text { sudamericano }\end{array}$ & 174 & 21,7 & & Profesional & 529 & 66,1 \\
\hline & Centroamérica & 122 & 15,2 & & Jubilado & 85 & 10,6 \\
\hline & Norteamérica & 189 & 23,6 & & Ama de casa & 38 & 4,8 \\
\hline & Europa & 217 & 27,1 & & Desempleado & 1 & ,1 \\
\hline & Asia & 55 & 6,9 & & & & \\
\hline
\end{tabular}

Fuente: Elaboración propia a partir de encuestas.

El análisis descriptivo de la percepción de congestión da cuenta de valores de tendencia central cercanos a 3 , lo que se interpreta como poco congestionado. Aunque un importante $22,6 \%$ de los encuestados percibe una extremada congestión, como se aprecia en el cuadro 3. 


\section{Cuadro 3 \\ FRECUENCIAS Y ESTADÍGRAFOS DE POSICIÓN SOBRE LA PERCEPCIÓN DE CONGESTIÓN}

\begin{tabular}{|lcc|}
\hline Percepción de congestión & Frecuencia & $\%$ \\
\hline 1-2 No tan congestionado & 12 & 1,5 \\
3-5 Un poco congestionado & 351 & 43,9 \\
6-7 Moderadamente congestionado & 256 & 32,0 \\
8-9 Extremadamente congestionado & 181 & 22,6 \\
Total & 800 & 100,0 \\
Media & & 2,76 \\
Mediana & & 3,00 \\
\hline
\end{tabular}

Fuente: Elaboración propia a partir de encuestas.

La prueba de Mann Whitney verificó que no hubo diferencias significativas respecto a la percepción de congestión y su valoración, entre los turistas que visitaron la Plaza de la Independencia y La Ronda. Así también se verificó la no existencia de diferencia significativa en la percepción de congestión entre grupos de turistas nacionales y extranjeros. Sin embargo se observó diferencia en la valoración de la congestión entre estos grupos, pues para los turistas nacionales la percepción fue mayoritariamente negativa. A continuación en el cuadro 4, se muestran los estadísticos de contraste calculados.

\section{Cuadro 4 \\ ESTADÍSTICOS DE CONTRASTE PARA PERCEPCIÓN Y VALORACIÓN DE CONGESTIÓN ENTRE GRUPOS}

\begin{tabular}{|l|l|l|l|}
\hline & Estadísticos de contraste & $\begin{array}{l}\text { Percepción de } \\
\text { Congestión }\end{array}$ & $\begin{array}{l}\text { Valoración de } \\
\text { congestión }\end{array}$ \\
\hline \multirow{2}{*}{$\begin{array}{l}\text { Entre grupos: plaza de la } \\
\text { Independencia y La Ronda }\end{array}$} & U de Mann-Whitney & 79264,000 & 76334,500 \\
\cline { 2 - 4 } & Sig. asintót. (bilateral) &, 809 &, 244 \\
\hline \multirow{4}{*}{$\begin{array}{l}\text { Entre grupos: nacionales y } \\
\text { extranjeros }\end{array}$} & Estadísticos de contraste & $\begin{array}{l}\text { Percepción de } \\
\text { Congestión }\end{array}$ & $\begin{array}{l}\text { Valoración de } \\
\text { congestión }\end{array}$ \\
\cline { 2 - 4 } & U de Mann-Whitney & 14898,500 & 9734,000 \\
\cline { 2 - 4 } & Sig. asintót. (bilateral) &, 213 &, 000 \\
\hline
\end{tabular}

Fuente: Elaboración propia a partir de encuestas.

Por otra parte, el análisis de componentes principales aplicado a la batería de motivaciones, arrojó un conjunto de 6 factores que pueden verse en el cuadro 5 , mientras que los resultados estadísticos se muestran en el cuadro 6 . 


\section{Cuadro 5}

FACTORES MOTIVACIONALES Y VARIABLES AGRUPADAS

\begin{tabular}{|c|c|c|}
\hline Factor & Denominación & Variables agrupadas \\
\hline Factor 1 & $\begin{array}{l}\text { Distracción, relajación y } \\
\text { capacitación }\end{array}$ & $\begin{array}{l}\text { Asistió a un festival o feria, mejora las capacidades, } \\
\text { relajamiento físico o mental }\end{array}$ \\
\hline Factor 2 & $\begin{array}{l}\text { Conocer el patrimonio } \\
\text { cultural }\end{array}$ & $\begin{array}{l}\text { Presentaciones culturales, ampliar conocimiento, } \\
\text { facilidades para compras, museos, designación patrimonio } \\
\text { de la humanidad, patrimonio e historia }\end{array}$ \\
\hline Factor 3 & Amigos y familia & $\begin{array}{l}\text { Pasar tiempo con amigos y familiares, visitar amigos y } \\
\text { familiares }\end{array}$ \\
\hline Factor 4 & $\begin{array}{l}\text { Tranquilidad, similitud, } \\
\text { cercanía y negocios }\end{array}$ & $\begin{array}{l}\text { Lugares de visita obligatoria, no tan lejos de mi lugar } \\
\text { habitual, negocios, tranquilidad en el centro histórico, } \\
\text { similitudes culturales con mi lugar habitual, experimentar } \\
\text { el entorno local }\end{array}$ \\
\hline Factor 5 & $\begin{array}{l}\text { Bares restaurantes y } \\
\text { nuevos amigos }\end{array}$ & Restaurantes, conocer nuevos amigos, bares \\
\hline Factor 6 & Conferencias & Conferencias \\
\hline
\end{tabular}

Fuente: Elaboración propia a partir de encuestas.

\section{Cuadro 6}

COMPONENTES PRINCIPALES PARA VARIABLES MOTIVACIONALES

\begin{tabular}{|lrrrrrr|}
\hline \multirow{2}{*}{ Motivación } & \multicolumn{7}{c|}{ Componente } \\
\cline { 2 - 7 } & 1 & 2 & 3 & 4 & \multicolumn{1}{c|}{5} & \multicolumn{1}{c|}{6} \\
\hline Conferencias &, 109 &, 217 &, 017 &, 281 &,- 049 &, 887 \\
Asistió a un festival o feria &, 593 &,- 091 &, 282 &, 097 &, 335 &, 443 \\
Presentaciones Culturales &, 154 &, 456 &, 296 &, 223 &, 190 &, 184 \\
Ampliar conocimiento &, 283 &, 785 &, 167 &,- 128 &, 095 &, 152 \\
Visito lugares de visita obligatoria &, 256 &, 330 &, 234 &, 449 &, 321 &, 142 \\
Facilidades para comprar &, 433 &, 514 &, 215 &, 063 &, 298 &, 125 \\
No llego tan lejos del lugar habitual &, 469 &,- 106 &, 269 &, 610 &, 003 &, 112 \\
Mejora las capacidades &, 813 &, 138 &, 116 &, 221 &, 205 &, 107 \\
Negocios &, 319 &, 355 &, 329 &, 473 &, 372 &, 081 \\
Experiencia de la historia y cultura local &, 351 &, 164 &, 091 &,- 318 &, 149 &, 077 \\
Restaurantes &, 244 &, 117 &, 402 &, 076 &, 733 &, 065 \\
Conoción nuevos amigos &, 194 &, 317 &,- 098 &,- 028 &, 594 &, 049 \\
Aprender más &, 316 &, 250 &, 096 &, 179 &,- 217 &, 047 \\
Pasar tiempo con amigos o familiares &, 327 &, 101 &, 840 &, 094 &,- 025 &, 022 \\
Nuevos lugares y cosas &, 276 &, 025 &, 314 &, 166 &, 184 &, 016 \\
Museos &, 165 &, 461 &, 357 &, 203 &, 232 &, 004 \\
Designación patrimonio de la humanidad &, 242 &, 560 &,- 049 &,- 170 &, 212 &,- 014 \\
\hline
\end{tabular}




\begin{tabular}{|lrlrlll|}
\hline \multirow{2}{*}{ Motivación } & \multicolumn{7}{c|}{ Componente } \\
\cline { 2 - 7 } & \multicolumn{1}{c|}{1} & 2 & 3 & 4 & \multicolumn{1}{c|}{5} & \multicolumn{1}{c|}{6} \\
\hline Visitó amigos o familiares &,- 052 &, 142 &, 912 &, 043 &, 252 &,- 027 \\
Bares &, 205 &, 166 &, 283 &, 106 &, 753 &,- 028 \\
Similitudes culturales de mi lugar habitual &, 162 &, 055 &,- 010 &, 727 &,- 103 &,- 046 \\
Experimento el entorno local &, 132 &, 420 &, 341 &, 436 &, 222 &,- 055 \\
Patrimonio &,- 124 &, 490 &, 022 &,- 016 &,- 043 &,- 089 \\
Se relajó físicamente o mentalmente &, 825 &, 062 &, 093 &, 163 &, 279 &,- 169 \\
Historia &,- 151 &, 409 &,- 016 &, 106 &, 115 &,- 261 \\
Repetiría su visita &, 057 &, 185 &, 036 &, 226 &,- 081 &,- 581 \\
\hline
\end{tabular}

Fuente: Elaboración propia a partir de encuestas.

En la misma línea, el análisis de componentes principales aplicado a la batería de emociones valorada, dio como resultado la conformación de dos factores o tipos de emociones: de interés, complacencia o contrariedad; y, de temor o decepción, como se aprecia en los cuadros 7 y 8 .

\section{Cuadro 7}

\section{FACTORES EMOCIONALES Y VARIABLES AGRUPADOS}

\begin{tabular}{|lll|}
\hline Factor & Denominación & Variables agrupadas \\
\hline Factor 1 & $\begin{array}{l}\text { Interés, complacencia o } \\
\text { contrariedad }\end{array}$ & Sorpresa, inspiración, coraje, alegría y admiración \\
Factor 2 & Temor o decepción & Miedo, tristeza y ansiedad \\
\hline
\end{tabular}

Fuente: Elaboración propia a partir de encuestas.

\section{Cuadro 8}

\section{COMPONENTES PRINCIPALES PARA VARIABLES EMOCIONALES}

\begin{tabular}{|c|c|c|}
\hline \multirow{2}{*}{ Emoción } & \multicolumn{2}{|c|}{ Componente } \\
\hline & 1 & 2 \\
\hline Emoción sorpresa & ,737 & 061 \\
\hline Emoción inspiración & 690 &,- 268 \\
\hline Emoción coraje & 670 & ,207 \\
\hline Emoción alegría & 655 &,- 146 \\
\hline Emoción admiración &, 599 &,- 096 \\
\hline Emoción miedo & 089 & 839 \\
\hline Emoción tristeza &,- 031 & 883 \\
\hline Emoción ansiedad &,- 251 &, 815 \\
\hline
\end{tabular}

Fuente: Elaboración propia a partir de encuestas. 
La aplicación del modelo de regresión logística a los factores generados y al resto de variables disponibles con el modelo, explica el $91 \%$ de la variabilidad de los datos, clasifica correctamente al $98 \%$ de los casos, y permite entender la percepción de congestión mediante la conversión binaria de la escala original expuesta en la siguiente fórmula:

$$
y=\frac{0=\text { no tan congesionado, ligera o moderadamente congestionado }}{1=\text { extremadamente congestionado }}
$$

Se ha considerado en este estudio que los tres niveles de congestión del numerador llevan implícito un grado aceptable de congestión, ya que los términos de la escala: "no tan", "ligero" y "moderado", refieren una percepción dentro de un límite imaginario que se fija el encuestado. Mientras que el nivel extremadamente congestionado lleva implícita la idea de haberse rebasado lo que se considera aceptable.

De acuerdo con esta conversión dicotómica, el análisis de regresión determina que 3 variables deben ser descartadas por cuanto sus coeficientes son estadísticamente iguales a cero (no significativos) y en ese sentido no muestran influencia en la percepción de congestión extrema por parte del turista. Tales variables son: tranquilidad, similitud, cercanía y negocios a nivel motivacional; nivel de estudios a nivel de perfil; y estadía a nivel de patrón de visita.

Así también los resultados demuestran que la percepción de congestión extrema tiene una valoración positiva para el turista. En otras palabras la congestión extrema no se percibe como negativa y de ahí se comprende que las emociones de temor y decepción se reduzcan en la medida en que la congestión se percibe más extrema.

En la misma línea se encuentra que no todos los comportamientos del visitante afectan la percepción de congestión. Tales son los casos de fumar, hacer ruido, pararse para tomar fotografías y obstaculizar el tráfico. Por el contrario, el tirar basura y chocar con otras personas influyen en la percepción de congestión extrema.

Por otra parte, la densidad de visitantes en los sitios fue bastante similar en los momentos de mayor afluencia: $\left(0,56\right.$ personas $/ \mathrm{m}^{2}$ en la Plaza de la Independencia y 0,58 personas/ $\mathrm{m}^{2}$ en la calle La Ronda). Los resultados indican que la densidad registrada no provoca que los turistas perciban los sitios fuera de un nivel aceptable de congestión. El cuadro 9 muestra los coeficientes de regresión calculados.

A nivel predictivo (considerando un R cuadrado de Cox y Snell de 0,60 y un R cuadrado de Nagelkerke de 0,91 ) el modelo indica que es más probable que el turista perciba los sitios extremadamente congestionados cuando las motivaciones están relacionadas con: la distracción, relajación y mejora de capacidades; el compartir tiempo con familia y amigos; el conocer el patrimonio cultural y el disfrute de bares, restaurantes y hacer nuevos amigos. Así también es más probable que se perciba extremada congestión al viajar en pareja o al observar a otros visitantes tirando basura. 
Cuadro 9

COEFICIENTES DE LA REGRESIÓN

\begin{tabular}{|l|c|c|c|c|c|c|}
\hline Variables & B & $\begin{array}{c}\text { Error } \\
\text { estándar }\end{array}$ & Wald & gl & Sig. & Exp(B) \\
\hline $\begin{array}{l}\text { Distracción, relajación y mejora de } \\
\text { capacidades }\end{array}$ & 5,554 & 1,021 & 29,611 & 1 & 0,000 & 258,263 \\
\hline Conocer el patrimonio cultural & 4,330 & 1,173 & 13,615 & 1 & 0,000 & 75,922 \\
\hline Amigos y familia & 5,666 & 1,067 & 28,190 & 1 & 0,000 & 288,924 \\
\hline Variables & B & $\begin{array}{c}\text { Error } \\
\text { estándar }\end{array}$ & Wald & gl & Sig. & Exp(B) \\
\hline $\begin{array}{l}\text { Tranquilidad, similitud, cercanía y } \\
\text { negocios }\end{array}$ & $-0,085$ & 0,668 & 0,016 & 1 & 0,899 & 0,919 \\
\hline Bares, restaurantes y nuevos amigos & 1,605 & 0,650 & 6,091 & 1 & 0,014 & 4,979 \\
\hline Conferencias & $-2,110$ & 0,591 & 12,756 & 1 & 0,000 & 0,121 \\
\hline Estudios superiores & 0,539 & 1,198 & 0,202 & 1 & 0,653 & 1,714 \\
\hline Estado civil & 2,308 & 0,975 & 5,606 & 1 & 0,018 & 10,050 \\
\hline Edad mayor o igual a 36 años & $-7,218$ & 1,690 & 18,230 & 1 & 0,000 & 0,001 \\
\hline Viaja solo o en pareja & $-6,256$ & 1,235 & 25,666 & 1 & 0,000 & 0,002 \\
\hline Estadía corta 1-3 días & $-1,648$ & 2,049 & 0,647 & 1 & 0,421 & 0,192 \\
\hline Congestión negativa & & 2,001 & 29,760 & 1 & 0,000 & 0,000 \\
\hline Interés, complacencia o contrariedad & 0,617 & 0,756 & 0,667 & 1 & 0,414 & 1,853 \\
\hline Temor o decepción & $-3,851$ & 1,086 & 12,565 & 1 & 0,000 & 0,021 \\
\hline La Ronda & $-0,864$ & 0,562 & 2,368 & 1 & 0,124 & 0,421 \\
\hline Fumar & 2,947 & 2,649 & 1,238 & 1 & 0,266 & 19,048 \\
\hline Hacer ruido & 0,234 & 1,977 & 0,014 & 1 & 0,906 & 1,263 \\
\hline Tomar fotografías & $-1,570$ & 2,174 & 0,522 & 1 & 0,470 & 0,208 \\
\hline Chocar con otras personas & $-7,569$ & 2,230 & 11,525 & 1 & 0,001 & 0,001 \\
\hline Tirar basura & 6,582 & 1,185 & 30,848 & 1 & 0,000 & 722,272 \\
\hline Obstaculizar el tráfico & $-2,420$ & 1,799 & 1,808 & 1 & 0,179 & 0,089 \\
\hline Densidad de personas por m² & $-1,488$ & 2,590 & 0,330 & 1 & 0,566 & 0,226 \\
\hline Constante & 3,707 & 5,342 & 0,482 & 1 & 0,488 & 40,726 \\
\hline
\end{tabular}

Fuente: Elaboración propia a partir de encuestas. 


\section{DISCUSIÓN}

El estudio no encuentra variación significativa entre la percepción de congestión extrema registrada entre turistas nacionales y extranjeros, lo cual corrobora las afirmaciones de Neuts y Nijkamp (2012) quienes notan que la mayoría de estudios refieren diferencias significativas en la percepción de congestión básicamente entre grupos de nacionalidad occidental y oriental.

Por otra parte como lo refiere Pedersen (2002), la percepción de congestión se relaciona no solo con la cantidad de personas presentes sino con sus pautas de comportamiento (Sun y Budruk, 2017). Así, este estudio confirma que el tirar basura y el choque entre personas influyen en la percepción de congestión extrema, mientras que no influyen otras conductas como fumar, hacer ruido, pararse para tomar fotografías u obstaculizar el tráfico; aunque en algunos estudios se asocia estas variables con la calidad de la experiencia del turista, como por ejemplo el ruido (Xue, Aili y Min, 2017). Esto podría explicarse en razón de tratarse de espacios públicos abiertos donde las personas están habituadas a encontrar este tipo de comportamientos.

A nivel emocional destaca la relación inversa entre la percepción de congestión extrema y los sentimientos de temor o decepción, que podría explicarse en el hecho de que parte de la expectativas de los turistas en las ciudades tienen que ver con apreciar el escenario urbano (Sassen y Roost, 1999) y esto incluye a la población local y su dinámica con el espacio público, por lo cual el encontrar numerosas personas en calles y plazas resulta atractivo para el turista, y en su defecto, encontrar poca presencia de gente puede ser causa de emociones como el temor o la decepción.

\section{CONCLUSIONES}

Con referencia a los momentos de más alta visitación en los sitios de interés turístico analizados, se concluye que para una densidad promedio de 0,57 personas $/ \mathrm{m}^{2}$, la mayoría de los turistas encuestados (77\%) perciben una congestión entre ligera y moderada.

El estudio no detecta diferencias significativas en la percepción de congestión entre personas extranjeras y nacionales, sin embargo si las encuentra respecto a cómo la valoran estos grupos. En ese sentido, para los turistas extranjeros el nivel de congestión percibido tiene una connotación positiva, notando que decrece la sensación de temor y decepción mientras más personas están presentes. En cambio, para el turista nacional el nivel de congestión percibido tiene un carácter negativo, distinguiéndose la influencia del comportamiento de otras personas, específicamente cuando tiran basura o impactan a otras mientras caminan.

Las motivaciones que caracterizan al grupo de turistas encuestados tienen que ver con: distracción y relajación; conferencias; familia y amigos; patrimonio cultural; bares y restaurantes; negocios y lugares tranquilos, cercanos y similares.

A nivel predictivo, el estudio indica que es menos probable que el turista perciba los sitios extremadamente congestionados, cuando le motivan los negocios y la visita a sitios próximos y parecidos a los que acostumbra. Por el contrario, es más probable que se perciban los sitios extremadamente congestionados al viajar en pareja y al observar a otros visitantes tirando basura. 
Desde el punto de vista de la gestión turística del patrimonio, los resultados son relativamente alentadores, pues aunque la mayoría de los turistas perciben un nivel aceptable de congestión, cerca de una cuarta parte $(22,6 \%)$ encuentran los sitios extremadamente congestionados. De éste porcentaje, aquellos que son nacionales lo perciben como algo negativo en su experiencia de visita.

En este sentido, las decisiones de los gestores del destino pueden también enriquecerse con información que proviene de los grupos minoritarios, teniendo presente las implicaciones del sello "Patrimonio de la Humanidad" y el papel de las visitas como vehículo para contribuir a la conservación y transmisión de su valor universal excepcional.

\section{LITERATURA CITADA}

ALMEIDA GARCÍA, F. (2006): «Tipología de visitante turístico y satisfacción de la experiencia turística en Santiago de Compostela», Baetica: Estudios de Arte, Geografía e Historia, vol. 28, pp. 231-258.

ASHWORD, G. (2012): «Do we understand urban tourism? », Tourism y Hospitality, vol. $1 \mathrm{n}^{\circ} 4$, pp. 1-2.

BARRER, D., HERNANDEZ, M. y BALBUENA, A. (2017): «Impacto de los festivales en el turismo patrimonial: el caso del Festival Internacional Cervantino», International Journal of Scientific Management and Tourism, vol. 3, n 3, pp. 47-66.

BENACH, N. y ALBET, A. (2018): «La gentrificación como una estrategia global», Papers 60, $\mathrm{n}^{\mathrm{O}} 17$, pp. 16-24.

BRITO, M. (2008): Las ciudades históricas como destinos patrimoniales: potencialidades y requisitos, Palma de Mallorca, Fundación Cátedra Iberoamericana.

BRIDA, J., RIAÑO, E. y ZAPATA, S. (2012): «Percepciones de los residentes acerca de los impactos del turismo de cruceros en la comunidad: un análisis factorial y de clústeres», Cuadernos de Turismo, n 29 , pp. 79-107

CAVAILlÈS, C., LAURENT, M., MAURÍN, S. y SÁNCHEZ HERNÁNDEZ, J.L. (2016): «Los turistas en el centro histórico de Salamanca: tránsito, percepción y (des) conocimiento», Cuadernos de Turismo, $\mathrm{n}^{\circ} 37$, pp. 37-67.

CORTÉS, T. (2002). Recuperación del patrimonio cultural urbano como recurso turístico. (Tesis doctoral). Universidad Complutense de Madrid. España.

COULOMB, R. (2008): «Sustentabilidad de la centralidad urbana e histórica, una reflexión desde el centro histórico de la ciudad de México», Quivera, Revista de Estudios Territoriales, vol. 10, n 2, pp. 29-49.

CRAVIDÃO, F., NOSSA, P., SANTOS, N., VIDAL, M., CROUS, N., AULET, S. y DALLARI, F. (2018): «Tourism at World Heritage Sites Opportunities and Challenges», en, Silvia De Ascaniis, Maria Gravari-Barbas and Lorenzo Cantoni (Eds.), Tourism Management at UNESCO World Heritage Sites, Lugano, Università della Svizzera italiana, pp. 23-30

DE LA CALLE VAQUERO, M. y GARCÍA, M. (2016): «Políticas locales de turismo en ciudades históricas españolas: Génesis, evolución y situación actual», Pasos, vol. 14, $\mathrm{n}^{\mathrm{o}} 3$, pp. 691-704 
DELGADILLO, V. (2015): «Patrimonio urbano, turismo y gentrificación». En V. Delgadillo, I. Díaz y L. Salinas (Coords.) Perspectivas del estudio de la gentrificación en México y América Latina. México, UNAM - Instituto de Geografía, pp. 113-133.

DONNELLY, M., VASKE, J., WHITTAKER, D. y SHELBY, B. (2000): «Toward an understanding of norm prevalence: A comparative analysis of 20 years of research», Environmental Management, vol. 25, n 4, pp. 403-414.

EAST, D., OSBORNE, P., KEMP, S. y WOODFINE, T. (2017): «Combining GPS y survey data improves understanding of visitor behavior», Tourism Management, vol. 61, pp. 307-320.

EMPRESA PÚBLICA METROPOLITANA DE GESTIÓN DE DESTINO TURISMO EPMGDT. (Agosto de 2015): Caracterización del turismo receptor en el DMQ: La demanda turística de viajeros no residentes a la ciudad de Quito $n^{\circ} 5$. Disponible en: https://www.quito-turismo.gob.ec/index.php/es/estadisticas/datos-turisticos-adicionales/category/11-gasto-y-perfil-del-turista?download=438:perfil-turista-receptor-en-eldmq-2014

LÓPEZ, F. (2005): «Quito, patrimonio mundial: 25 años después resumen de un proyecto integral de gestión». En M. Villamor y E. Villamor. Primeras Jornadas de Patrimonio Cultural en América Latina. Madrid, Areté Documenta, Revista de la Asociación Española de Gestores de Patrimonio Cultural, pp. 143-164

MUNICIPIO DEL DISTRITO METROPOLITANO DE QUITO (2003): Plan Especial del Centro Histórico de Quito. Disponible en: http://www.flacsoandes.edu.ec/libros/ digital/39693.pdf

MUNICIPIO DEL DISTRITO METROPOLITANO DE QUITO. (2011): Plan de desarrollo 2012 - 2022. Disponible en: http://www.quito.gob.ec/documents/rendicion_cuentas/AZC/Articulacion_politicas_publicas/PLAN_ORDENAMIENTO_ TERRITORIAL2012.pdf

FLEISHMAN, L., FEITELSON, E., y SALOMON, I. (2004): «The role of cultural and demographic diversity in crowding perception: evidence from nature reserves in Israel», Tourism Analysis, vol. 9, n 1-2, pp. 23-40

GALÍ ESPELT, N. (2008): «El comportamiento turístico en las ciudades monumentales: el caso de Girona». En M. Troitiño, J. García y M. García (Coords.) Destinos turísticos: viejos problemas, ¿nuevas soluciones? Cuenca, Ediciones de la Universidad de Castilla-La Mancha, pp. 99 -115.

GARCÍA, M. y DE LA CALLE, M. (2012). «Capacidad de carga en grandes recursos turístico-culturales», Anales de Geografía, vol. 32, n 2, pp. 253-274

GARCÍA, M., DE LA CALLE, M. y MINGUEZ, C. (2011): «Capacidad de carga turística y espacios patrimoniales. Aproximación a la estimación de la capacidad de carga del conjunto arqueológico de Carmona (Sevilla, España)». Boletín de la Asociación de Geógrafos Españoles, no 57, pp. 219-241

GONZÁLEZ, J. (2013). «Problemáticas urbanas en los enclaves turísticos: turismo como estrategia para el ordenamiento urbano y territorial», Revista Bitácora Urbano Territorial, vol. $22, \mathrm{n}^{\mathrm{o}} 1$, pp. 138-140 
HERNÁNDEZ, F., VARGAS, J. y AGUILAR, J. (2015). «El efecto emocional de una experiencia turística: el mercado de artesanías de Oaxaca, México», Cathedra et Scientia International Journal, vol. 1, n 1, pp. 147-154

HIERNAUX, D. y GONZÁLEZ, I. (2015): «Turismo y gentrificación: pistas teóricas sobre una articulación», Revista de Geografía Norte Grande, n ${ }^{\circ}$ 58, pp. 55-70.

HOSANY, S. y GILBERT, D. (2010). «Measuring Tourists' Emotional Experiences toward Hedonic Holiday Destinations», Journal of Travel Research, vol. 49, n 4, pp. 513-526.

HUANG, Y., SCOTT, N., DING, P. y CHENG, D. (2012): «Impression of Liusanjie: Effect of Mood on Experience and Satisfaction», International Journal of Tourism Research, vol. $14, \mathrm{n}^{\circ} 1$, pp. 91-102.

HUI, M. y BATESON, J. (1991): «Perceived control and the effects of crowding and consumer choice on the service experience», The Journal of Consumer Research, vol. $18, \mathrm{n}^{\circ} 2$, pp. 174-184.

JIMÉNEZ, P. y AQUINO, F. (2012): «Propuesta de un modelo de competitividad de destinos turísticos», Estudios y Perspectivas en Turismo, vol. 21, no 4, pp. 977-995

LUQUE, A., GÓMEZ, M. y PELÁEZ, M. (2018): «Starting to enjoy nature in Mediterranean mountains: Crowding perception and satisfaction», Tourism Management Perspectives, vol. 25, pp. 93-103

MORALES, E., y LANQUAR, R. (2014): «El futuro turístico de una ciudad patrimonio de la humanidad: Córdoba 2031», Tourism y Management Studies, vol. 10, n 2, pp. 7-16

MORENO, R.; PÉREZ, J.; OYARCE, F.y y LÓPEZ, T. (2016): «Factores de interés de un destino patrimonio de la humanidad: El caso de Valparaíso-Chile», Estudios y Perspectivas en Turismo, vol. 25, n 3, pp. 360-374

MOYLE, B., SCHERRER, P., WEILER, B., WILSON, E., CALDICOTT, R.y y NIELSEN, N. (2017): «Assessing preferences of potential visitors for nature-based experiences in protected areas», Tourism Management, vol. 62, pp. 29-41

NAOI, T., AIREY, D., IIJIMA, S., y NIININEN, O. (2007): «Towards a theory of visitors' evaluation of historical districts as tourism destinations: Frameworks and methods», Journal of Business Research, vol. 60, n 4, pp. 396-400

NEUTS, B.; NIJKAMP, P. y VAN LEEUWEN, E. (2012): «Crowding externalities from tourist use of urban space», Tourism Economics, vol. 18, n 3 , pp. 649-670

NEUTS, B. y NIJKAMP, O. (2012): «Tourist crowding perception and acceptability in cities: An Applied Modelling Study on Bruges», Annals of Tourism Research, vol. 39, $\mathrm{n}^{\circ} 4$, pp. 2133-2153

NICKERSON, N. (2016): «"What We Know about Crowding and Visitor Experiences»», Institute for Tourism and Recreation Research Publications. Paper 340. Disponible en : http://scholarworks.umt.edu/itrr_pubs/340

WORLD TOURISM ORGANIZATION. (2012): Global Report on City Tourism (AM Report $n^{\circ} 6$ ). Disponible en: http://cf.cdn.unwto.org/sites/all/files/pdf/am6_city_platma.pdf

PEDERSEN, A. (2002): Managing Tourism at World Heritage Sites: a Practical Manual for World Heritage Site Managers. World Heritage Centre, UNESCO. Disponible en: http://unesdoc.unesco.org/images/0012/001286/128679s.pdf 
PLUTCHIK, R. (1980): Emotion: A Psychoevolutionary Synthesis. New York, Harper and Row.

PULIDO, J. y NAVARRO, U. (2014): «Identificación de ítems para medir las experiencias del turista en destino», Cultur, Revista de Cultura e Turismo, vol. 8, n 1, pp. 4-34

QIAN JIN, Q., HU, H. y KAVAN, P. (2016): «Factors Influencing Perceived Crowding of Tourists and Sustainable Tourism Destination Management», Sustainability, vol. 8, $\mathrm{n}^{\mathrm{o}} 10, \mathrm{p} .976$.

RITCHIE, J. y CROUCH, G. (2003): The competitive destination: a sustainable tourism perspective. Wallingford, CABI.

RUIZ, A. (2012): Guanajuato (México): dinámica y estructura de un destino turístico patrimonio de la humanidad. (Tesis doctoral). Universidad Complutense de Madrid. España.

RUSSO, A. (2002): «The "vicious circle" of tourism development in heritage cities», Annals of Tourism Research, vol. 29, n 1, pp. 165-182.

SANTANA-JIMÉNEZ, Y. y HERNÁNDEZ, J. (2011): «Estimating the effect of overcrowding on tourist attraction: The case of Canary Islands», Tourism Management, vol. 32, no 2 , pp. 415-425

SALMERÓN, P. (2009): «Reflexión sobre una experiencia». En P. Salmerón Escobar y N. Sanz (Eds.), El paisaje histórico urbano en las ciudades históricas Patrimonio de la Humanidad. Indicadores para su conservación y gestión. Sevilla, Instituto Andaluz del Patrimonio Histórico. pp. 8-21

SASSEN, S., y ROOST, F. (1999): «The city: strategic site for the global entertainment industry». En D. Judd y S. Fainstein (Eds.), The tourist city, New Haven, Yale University Press, pp. 143-154.

SHELBY, B., VASKE, J. y HEBERLEIN, T. (1989): «Comparative analysis of crowding in multiple locations: Results from fifteen years of research», Leisure Sciences, vol. $11, n^{\circ} 4$, pp. 269-291

SHIA, B., ZHAO, J., y CHEN, P.J. (2017): «Exploring urban tourism crowding in Shanghai via crowdsourcing geospatial data». Current Issues in Tourism, vol. 20, $\mathrm{n}^{\mathrm{o}} 11$, pp. 1.186-1.209.

SUN, Y.Y. y BUDRUK, M. (2017): «The moderating effect of nationality on crowding perception, its antecedents, and coping behaviours: A study of an urban heritage site in Taiwan», Current Issues in Tourism, vol. 20, $\mathrm{n}^{\circ}$ 12, pp. 1.246-1.264.

TORRES, P. y VILLACÍS, C. (2016): «Las ciudades del patrimonio mundial como destinos altamente posicionados». Retos Turísticos, vol. 15, n 2, pp.1-14.

TROITIÑO, M.A. (1998): «Turismo y desarrollo sostenible en ciudades históricas». Ería, $\mathrm{n}^{\mathrm{o}} 47$, pp. 221-227.

VASKE, J. y DONNELLY, M. (2002): Generalizing the encounter-norm-crowding relationship, Leisure Sciences, vol. 24, n 3-4, pp. 255-269.

XUE, H., AILI, L. y MIN, L. (2017): «Noise Perception and Its Effects on Tourists> Satisfaction: A Case Study of Nanluoguxiang Lane in Beijing», INTER-NOISE and NOISECON Congress and Conference Proceedings, InterNoise 17, Hong Kong, pp. 136-146.

ZHANG, L. y CHUNG, S. (2015): «Assessing the social carrying capacity of diving sites in Mabul Island», Environment Management, vol. 56, n 6, pp. 1.467-1.477. 